\title{
TeV $\gamma$-ray astronomy with ground-based air-shower arrays
}

\author{
Miguel A. Mostafáa \\ Department of Physics, The Pennsylvania State University, University Park, 16802 PA, USA
}

\begin{abstract}
The $\mathrm{TeV}$ energy band is a very exciting window into the origin of high energy cosmic radiation, particle acceleration, and the annihilation of dark matter particles. Above a few hundred $\mathrm{GeV}$, ground-based experiments of very large effective areas open a new domain to study extragalactic sources at intermediate redshifts, galaxy clusters, gamma ray bursts, AGN and their flaring states, extended sources and galactic diffuse emission, and to indirect searches for dark matter. In particular, ground arrays of particle detectors -that operate with high duty cycles and large fields of view- can extend to multi- $\mathrm{TeV}$ energies the measurements made with experiments on satellites, and complement the observations done with air Cherenkov telescopes on the ground. Key science goals of ground arrays include performing unbiased all-sky surveys, monitoring of transient events from known (and unknown) sources, and detecting extended regions of diffuse emission. In this paper, the status and most recent results from ARGO-YBJ, Tibet AS, HAWC, and LHAASO are presented.
\end{abstract}

\section{Introduction}

The measurement of the spatial and energy distribution of very high energy $\gamma$ rays is an essential tool to identify and understand the cosmic accelerators of the highest energy particles. On the other hand, the time variability and energy spectra of the $\gamma$-ray emission can be used to study the environment of these accelerators and the acceleration mechanisms. Ground-based arrays of particle detectors play a key role in very high energy $\gamma$-ray astronomy due to their large active area $\left(>10^{4} \mathrm{~m}^{2}\right)$, high duty cycle (usually $>90 \%$ ), and large field of view ( $\sim 2 \mathrm{sr}$ ). Thus, ground arrays can extend the spectra of known $\gamma$-ray sources beyond the reach of space-based detectors, characterize spatially-extended sources that are difficult to be observed with telescopes, and trigger multi-wavelength observations of transient events without the need to steer.

The experimental techniques used for each ground array are described in Sect. 2. The most recent results and the current status of ARGO-YBJ, Tibet AS, HAWC, and LHAASO are presented in Sect. 3. The outlook of $\mathrm{TeV} \gamma$-ray astronomy with ground-based arrays is summarized in Sect. 4.

ae-mail: miguel@psu.edu

(C) The Authors, published by EDP Sciences. This is an Open Access article distributed under the terms of the Creative Commons Attribution License 4.0 (http://creativecommons.org/licenses/by/4.0/). 


\section{Detection techniques}

\subsection{Resistive plate chambers}

The Astrophysical Radiation with Ground-based Observatory at YangBaJing (ARGO-YBJ) experiment [1] is located at 4,300 m above sea level at the Yangbajing Cosmic Ray Observatory in Tibet, China (90.5 east, $30.1^{\circ}$ north), and was designed for very-high energy $\gamma$-ray astronomy and cosmic-ray observations.

The ARGO-YBJ experiment is a full coverage Resistive Plate Chamber (RPC) array. The array consists of a single layer of 153 "clusters" $(7.6 \mathrm{~m} \times 5.7 \mathrm{~m})$ of $12 \mathrm{RPCs}$ each. To collect the induced charges when particles pass through the layer, RPCs are operated in streamer mode with a gas mixture of $\mathrm{C}_{2} \mathrm{H}_{2} \mathrm{~F}_{4} / \mathrm{Ar} / i-\mathrm{C}_{4} \mathrm{H}_{10}=75 / 15 / 10$ and a voltage of $7.2 \mathrm{kV}$ applied to the $2 \mathrm{~mm}$ gas gap. To provide a larger dynamic range for particle counting, each RPC is read out in 10 pads of 8 strips $(62 \mathrm{~cm} \times 7 \mathrm{~cm})$ each. Signals from the strips are digitized by 18,360 time-to-digital converters (TDCs) to provide timing and position information of the particles. The signals from the strips of a given pad are sent to the same channel of the TDC. The arrival times of the particles are measured with a resolution of $\sim 1.8 \mathrm{~ns}$ [1]. The central 130 clusters form the central carpet with an active area of $\sim 92 \%$ of the $74 \mathrm{~m} \times 78 \mathrm{~m}$, and began recording data in July 2006. The surrounding 23 additional (partially instrumented) clusters (a.k.a. the "guard ring") were merged into the DAQ stream in November 2007. To extend the dynamical range up to PeV energies, the analog charge of each RPC is read out with two large pads $(1.4 \mathrm{~m} \times 1.2 \mathrm{~m})$, each one covering half a chamber. These larger pads (also called "big pads") collect the total charge deposited by the particles hitting the detector [2]. The total area of the array is $110 \mathrm{~m} \times 100 \mathrm{~m}$. The installation of the RPCs started in 2001, and the central carpet was commissioned in June 2006. Data taking with the full detector started in November 2007, and ended in February 2013. ARGO-YBJ has collected over $5 \times$ $10^{11}$ events in this period.

The ARGO-YBJ detector is operated by requiring the coincidence of at least 20 triggered pads $\left(N_{\text {pad }}\right)$ within $420 \mathrm{~ns}$ on the entire detector. The time (and location) of each triggered pad within a window of $2 \mu$ s are recorded. The trigger rate of ARGO-YBJ is $3.5 \mathrm{kHz}$ with a dead time of $4 \%$ and an average duty-cycle higher than $86 \%$. The shower core is estimated using a maximum likelihood method for fitting the lateral density distribution of the shower using a modified Nishimura-Kamata-Greisen (NKG) function. The core resolution (68\% containment) is better than $10 \mathrm{~m}$ for events with $N_{\text {pad }}>100$. The arrival direction is reconstructed using a least-squares method assuming a conical shape of the shower front. The angular resolution for $\gamma$ rays ranges from $1.7^{\circ}$ to $0.2^{\circ}$, depending on the size of the shower quantified by the number of hit pads [3].

The effective area of the ARGO-YBJ array was estimated using Monte Carlo simulations [3], and found to be $\sim 100 \mathrm{~m}^{2}$ at $100 \mathrm{GeV}$ and $\sim 10,000 \mathrm{~m}^{2}$ above $1 \mathrm{TeV}$ for a zenith angle of $10^{\circ}$.

The number of triggered pads $N_{\text {pad }}$ is the observable commonly used to infer the primary energy. But due to the large shower fluctuations and the dependance of the shower size with the position of the shower core with respect to the detector center, $N_{\text {pad }}$ does not actually allow a precise determination of the primary energy on an event-by-event basis. Instead, the energy spectrum of a $\gamma$-ray source is determined from the comparison of the observed distribution of $N_{\text {pad }}$ with simulations.

\subsection{Scintillators}

The Tibet Air-Shower (AS) experiment [4], also located at the Yangbajing Cosmic Ray Observatory in China, is an array of 761 scintillation counters placed on a lattice of $7.5 \mathrm{~m}$ 
spacing, and 28 scintillation counters on a lattice of $15 \mathrm{~m}$ spacing. The total area cover by the array is almost $37,000 \mathrm{~m}^{2}$. Each counter has a plate of plastic scintillator, $0.5 \mathrm{~m}^{2}$ in area and $3 \mathrm{~cm}$ thick, and a 2" PMT. Additionally, there are burst detectors (BDs) at the center of the array to measure the core region of the air showers. Each BD is composed of a plate of plastic scintillator $(160 \mathrm{~cm} \times 50 \mathrm{~cm} \times 2 \mathrm{~cm})$ and 4 photodiodes attached to each corner of the plate. They detect the high-energy particles in the core of the showers by converting them into electromagnetic cascade showers in a lead target. The total thickness of the lead plates is $3.5 \mathrm{~cm}$ ( 7 radiation lengths). The location of the burst can then be determined with a spatial resolution of $10 \mathrm{~cm}$. A total of $100 \mathrm{BDs}$ cover an area of $80 \mathrm{~m}^{2}$ near the center of the air-shower array. The Tibet AS array has been taking data in this hybrid configuration since 1999.

The time and charge information from each triggered PMT are recorded to determine the direction and energy of the air shower. The energy of the showers is estimated from the shower size at ground, which is determined from the lateral charge distribution using an NKG-like function. The energy resolution is $\sim 20 \%$ around the knee. Arrival directions are determined using the timing information from the counters and assuming a conical shower front. The angular resolution, estimated with the Moon's shadow, is $0.2^{\circ}$ for energies above $100 \mathrm{TeV}$. The trigger rate is $\sim 1.5 \mathrm{kHz}$, and the threshold energy is $\sim 3 \mathrm{TeV}$.

\subsection{Water Cherenkov detectors}

The High Altitude Water Cherenkov (HAWC) observatory [5] is located on a $200 \mathrm{~m} \times 450 \mathrm{~m}$ plateau at 4,100 $\mathrm{m}$ above sea level inside the Pico de Orizaba national park in Mexico. The design of HAWC is based on the experience with the Milagro experiment [6]. Milagro was the first generation of $\gamma$-ray detectors using the water Cherenkov technology, and consisted of a big pond with a double layer of PMTs at the bottom. Instead, HAWC is an array of individual water Cherenkov detectors (WCDs) with a single deep layer of PMTs with a wider separation. This configuration provides a larger active area for the same photo-cathode area, and optical isolation for the WCDs.

The HAWC array will consist of 300 WCDs densely-spaced over an area of 22,000 $\mathrm{m}^{2}$. The structural support of the WCDs is provided by corrugated steel tanks of $7.3 \mathrm{~m}$ diameter. There is a light-tight plastic bladder inside each tank, filled with almost 200,000 liters of ultrapure water up to a height of $4.5 \mathrm{~m}$. There are four upward-facing, baffled PMTs anchored to the bottom of each WCD. Three of them are 8" PMTs, previously used in Milagro, and located $6 \mathrm{ft}$ from the center of the tank. The fourth, central tube is a high quantum-efficiency 10" PMT designed to increase the sensitivity of HAWC to air-showers with energies below $1 \mathrm{TeV}$.

The design of HAWC combines the water Cherenkov technique with a high altitude site. The higher altitude, the increased active area, and the optical isolation of the PMTs lead to a better angular and energy resolution, improved $\gamma /$ hadron separation, and an order of magnitude increase in sensitivity with respect to Milagro. The full HAWC array will observed a $5 \sigma$ signal from the Crab Nebula in a single 4-hr transit while maintaining this sensitivity over 2 sr. Charged cosmic rays are discriminated from $\gamma$ rays by observing large energy depositions far $(>40 \mathrm{~m})$ from the shower core. The cosmic-ray rejection is estimated from air-shower simulations, and found to be better than $99 \%$ for energies above $\sim 3 \mathrm{TeV}$. As expected, the rejection efficiency improves with energy. The arrival direction of an air shower is determined from the timing information from the PMTs, and estimated (also from simulations) to be $0.1^{\circ}$ for air showers with energies above $10 \mathrm{TeV}$. 


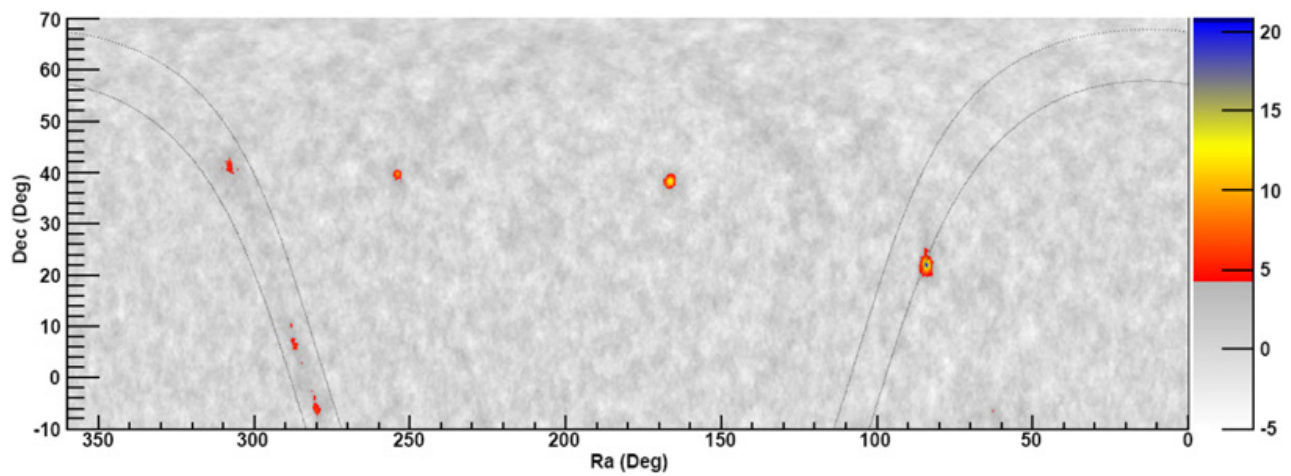

Figure 1. Figure 3 from Ref. [3].Survey of the Northern sky in $\gamma$-rays with the ARGO-YBJ experiment in the energy range from $10 \mathrm{GeV}$ to $100 \mathrm{TeV}$. The six sources observed with a statistical significance greater than 5 s.d. are consistent in position with the Crab Nebula, the blazars Mrk 421 and Mrk 501, MGRO J2031+41 (and TeV J2032+4130), HESS J1841-055, and HESS J1908+063. The significances of the excesses, in terms of standard deviations, are shown by the color scale on the right side. The two dotted lines indicate the Galactic latitudes $b= \pm 5^{\circ}$.

\section{Status and results}

\subsection{ARGO-YBJ}

The ARGO-YBJ detector carpet is connected to two DAQ systems that work independently. In the shower DAQ mode, the location and timing of each detected particle is recorded for each event. This mode allows the reconstruction of the arrival direction and of the lateral distribution for each shower. The threshold for this mode is $E_{\mathrm{th}} \approx 300 \mathrm{GeV}$. Instead, in the scaler DAQ mode the counting rate of each cluster is measured every $0.5 \mathrm{~s}$. Thus, in this mode there is no information on either the charge distribution of the detected secondary particles on the ground, or the arrival direction of the shower. The threshold for the scaler mode is lower, $E_{\mathrm{th}} \approx 1 \mathrm{GeV}$.

The ARGO-YBJ collaboration performed a survey of the Northern sky $\left(-10^{\circ}<\delta<\right.$ $70^{\circ}$ ) to search for steady VHE $\gamma$-ray emitters using more than five years of data. This corresponds to an integrated sensitivity ranging from 0.24 to $\sim 1$ Crab units (depending on the declination). They have detected six sources with a statistical significance greater than 5 standard deviations (s.d.) [3]. These sources, shown in Fig. 1, are consistent in position with well known $\mathrm{TeV} \gamma$-ray emitters: the Crab Nebula, the blazars Mrk 421 and Mrk 501, MGRO J2031+41 (and TeV J2032+4130), HESS J1841-055, and HESS J1908+063. They also reported evidence for possible $\mathrm{TeV}$ emission from five directions. (The locations of the excesses with significance greater than 4.0 s.d.) Two of these five excesses are not associated with any known counterpart and one of them is coincident in position with a high-mass $X$-ray binary.

The spectral energy distribution (SED) of the Crab Nebula (shown in Fig. 2) is fitted in the energy range from $0.1 \mathrm{TeV}$ to $35 \mathrm{TeV}$. The spectral index of this fit is $-2.62 \pm 0.06$ (stat). This SED derived from the ARGO-YBJ data is in agreement, within uncertainties, with the results obtained by other experiments, e.g. HEGRA, H.E.S.S., MAGIC, and Tibet AS [7-10]. The comparison is also shown in figure 2. (Only statistical uncertainties are shown.) The integral flux of this spectrum is $1.85 \times 10^{-11} \mathrm{~cm}^{-2} \mathrm{~s}^{-1}\left(5.69 \times 10^{-11} \mathrm{~cm}^{-2} \mathrm{~s}^{-1}\right)$ above $1 \mathrm{TeV}(500 \mathrm{GeV})$. The systematic uncertainties on the flux for point sources are less than $30 \%$ [11]. 


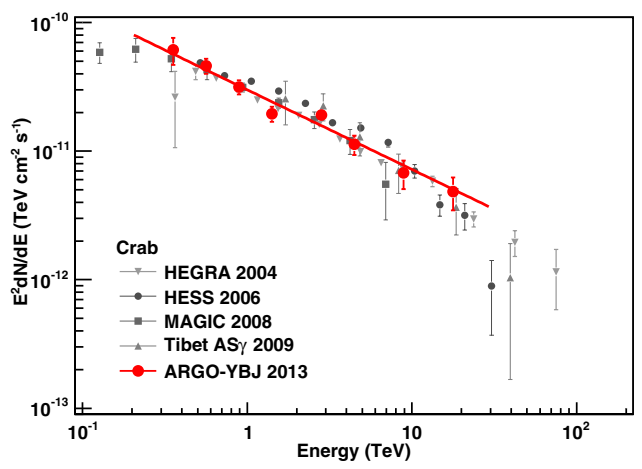

Figure 2. Figure 5 from Ref. [3]. Spectral energy distribution of the Crab Nebula measured with the ARGO-YBJ experiment, and comparison with the measurements from HEGRA, H.E.S.S., MAGIC, and Tibet AS [7-10]. The (red) line is the best fit to the ARGO-YBJ data using a power-law function. The spectral index is -2.6 . (Only statistical uncertainties are shown.)
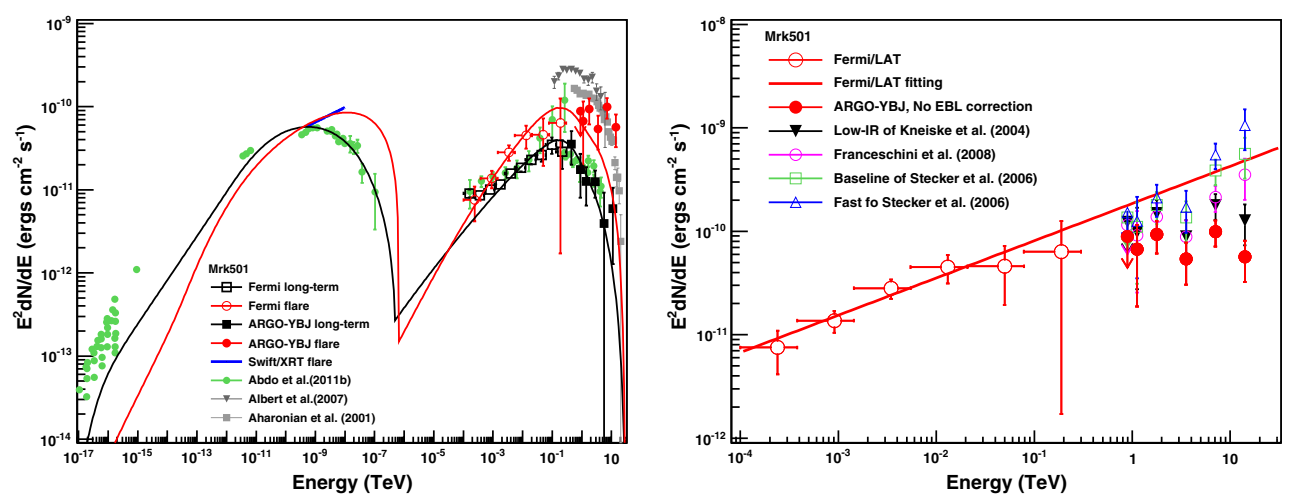

Figure 3. Left: spectral energy distribution of Mrk 501. (Fig. 6 from Ref. [13].) The solid black (red) line shows the best fit to the long-term (flaring) data using a one-zone SSC model. The best-fit parameters can be found in Ref. [13]. Right: spectra of Mrk 501 during the flaring period between October 2011 and April 2012. (Fig. 5 from Ref. [13].) The spectrum below $300 \mathrm{GeV}$ is from the Fermi-LAT. Data from ARGO-YBJ has been corrected using four EBL models. Details can be found in Ref. [13].

The ARGO-YBJ collaboration also performed a 4-year long continuous monitoring of Mrk 501 between August 2008 and April 2012 [13]. Both the flux (0.3 Crab units above $1 \mathrm{TeV})$ and the spectral index (-2.6) measured with ARGO-YBJ between $450 \mathrm{GeV}$ and $10 \mathrm{TeV}$ are consistent with those obtained with other experiments. The fit to the long-term averaged SED of Mrk 501 over a very wide energy range (combining the radio-to-TeV campaign during the first 480 days of Fermi operation presented in Ref. [12] with the ARGOYBJ data above $300 \mathrm{GeV}$ ) favors the $\gamma$-ray emission due to the inverse Compton scattering of the synchrotron radiation (synchrotron self-Compton, SSC) from the same population of relativistic electrons [13]. (The electron energy distribution is parameterized using a single power-law function with an exponential cutoff at its high energy end.) The best fit to the average broadband spectrum of Mrk 501 (from radio to $\mathrm{TeV}$ ) in the framework of the onezone SSC model is the black solid line in the left panel of Fig. 3.

A significant $(>6 \sigma)$ excess in the event rate was detected by ARGO-YBJ in the direction of Mrk 501 between October 17 and November 22, 2011. This bright flaring episode 
represented a large $(\times 7)$ increase in the $\gamma$-ray flux observed above $1 \mathrm{TeV}$ with respect to the steady-state emission [13]. The hardness of the spectrum observed during the flare (shown in the right panel of Fig. 3) challenges the one-zone SSC model that is in good agreement with the long-term spectrum.

Previously, the ARGO-YBJ collaboration had also reported a long-term continuous monitoring of Mrk 421. The observation time reported in Ref. [14] (December, 2007 to February, 2010) covered both active and quiet phases. The $\gamma$-ray flux observed with the ARGO-YBJ experiment shows a good long-term correlation with the satellite-borne $X$-ray data. They also found that all the large $X$-ray flares have $\gamma$-ray counterparts in the ARGOYBJ data. The temporal and spectral analysis of the Mrk 421 data also strongly favors the hypothesis that $\gamma$-rays and $X$-rays have a common origin as assumed in the SSC radiative mechanism.

The ARGO-YBJ collaboration had also reported (using data up to August, 2011 [11]) the observation of $\mathrm{TeV} \gamma$-rays from a large part of the Cygnus region, which indicates a possible diffuse $\gamma$-ray emission. A statistical significant $(>6 \sigma)$ excess was observed from the direction of MGRO J2031+41. The measured flux is consistent with the Milagro observation, but higher than the flux observed with MAGIC. No evidence of an emission above $3 \sigma$ is found at the location of MGRO J2019+37 (inside the Cyg OB1 region) even considering extended sources. MGRO J2019+37, detected with the Milagro experiment at a median energy of $20 \mathrm{TeV}$ [15], is the second most significant source in the Milagro data set after the Crab Nebula. The upper limits (90\% c.l.) derived for MGRO J2019+37 do not confirm the spectrum determined with Milagro at energies below $5 \mathrm{TeV}$, and the exposure of ARGOYBJ above $5 \mathrm{TeV}$ is too low to reach a firm conclusion. Because the two observations were performed five years apart, variability in the VHE $\gamma$-ray flux of this source has been proposed as an explanation of this discrepancy. On the other hand, this would be in direct conflict with the identification of this source as a PWN. It is important to note that the upper limits from ARGO-YBJ are consistent with a new fit to the Milagro data assuming a power law with an exponential cutoff $(\sim 30 \mathrm{TeV})$ and a hard spectral index $(-2.0)[16]$.

A more recent analysis of the ARGO-YBJ full data set (November, 2007 to January, 2013) [17] suggests that the location of the extended TeV $\gamma$-ray source ARGO J2031+4157 (or MGRO J2031+41) is consistent with the Cygnus Cocoon discovered by Fermi-LAT at $\mathrm{GeV}$ energies in the Cygnus superbubble [18]. The significance map around the Cygnus Cocoon as observed in the ARGO-YBJ full data set is shown in the left panel of Fig. 4. The most statistically significant $(>6 \sigma$ ) excess in this map corresponds to the position of ARGO J2031+4157, and has a similar angular size $\left(\sim 2^{\circ}\right)$ than the Cygnus Cocoon as measured with Fermi-LAT. After subtracting the contribution of the overlapping TeV sources (details can be found in Ref. [17]), they find a differential energy spectrum with a spectral index $-2.6 \pm 0.3$ in the energy range $0.2-10 \mathrm{TeV}$. The spectral measurements with Fermi-LAT, ARGO-YBJ, and Milagro are shown in the right panel of Fig. 4. The flux determined with ARGO-YBJ appears consistent with the extrapolation of the spectrum of the Cygnus Cocoon as measured with Fermi-LAT in the $1-100 \mathrm{GeV}$ energy range. The similarity of the angular extensions and the consistency of the spectral measurements from $1 \mathrm{GeV}$ to $10 \mathrm{TeV}$ suggest that the $\mathrm{TeV}$ emission from ARGO J2031+4157 may be identified as the counterpart of the Cygnus Cocoon observed at GeV energies. The combined data from Fermi-LAT and ARGO-YBJ can be fitted with the power law function with a spectral index $-2.16 \pm 0.04$ as shown by the dot-dashed line in the right panel of Fig. 4. (The Milagro data points refer to the source MGRO J2031+41 from Refs. [19-21].) The slope of the spectral measurements from $1 \mathrm{GeV}$ to $10 \mathrm{TeV}$ indicates that the particle acceleration inside a superbubble is similar to that in a Supernova Remnant. Assuming a hadronic model where the primary proton spectrum follows 

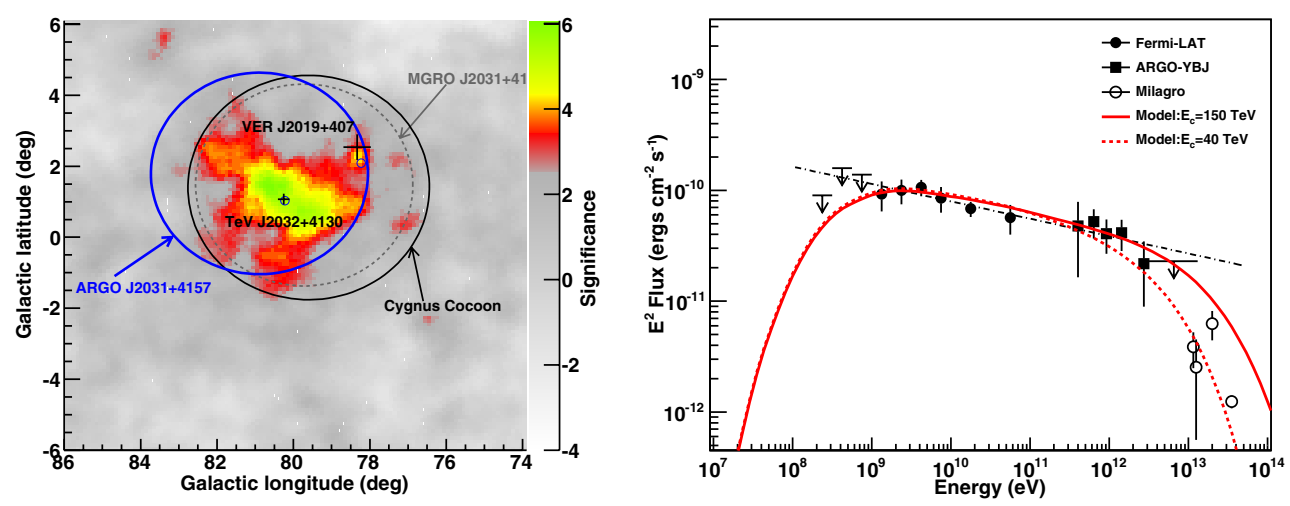

Figure 4. Left: significance map around PSR 2032+4127 in the Cygnus region as observed using ARGO-YBJ data. (Fig. 1 from Ref. [17].) The positions of other known TeV sources and of the Cygnus Cocoon are also marked. Right: spectral energy distribution of the Cygnus Cocoon region as measured with different experiments. (Fig. 3 from Ref. [17].) The measurements at 12, 20 and $35 \mathrm{TeV}$ are reported by the Milagro collaboration for MGRO J2031+41 in Refs. [19-21]. (The lower data point at $12 \mathrm{TeV}$ is the flux in Milagro after subtracting the contribution from TeV J2032+4130 [18].) The dot-dashed line corresponds to the best fit to the combined Fermi-LAT and ARGO-YBJ data assuming a simple powerlaw spectrum. The red lines represent hadronic models with two proton cutoff energies (at $150 \mathrm{TeV}$ and $40 \mathrm{TeV})$.

a power law (with the same spectral index of $\gamma$-rays) and an exponential cutoff, the flux upper limits from Fermi-LAT and ARGO-YBJ suggest a change of slope below $\sim 1 \mathrm{GeV}$ and a cutoff above $\sim 10 \mathrm{TeV}$.

Other important contributions from the ARGO-YBJ collaboration include the detection of diffuse $\gamma$ rays from the Galactic plane (journal paper in preparation) ${ }^{1}$, and the fluence upper limits to the emission from Gamma Ray Bursts (GRBs) in the interesting energy range between 1 and $100 \mathrm{GeV}$ [23]. In a more recent analysis of the ARGO-YBJ data set up to January, 2013, no significant excess coincident with satellite detection was found for 206 GRBs inside the ARGO-YBJ field of view, the largest sample ever investigated with a groundbased detector.

\subsection{Tibet AS}

Using the Tibet-III array, that was completed in 1999, the Tibet AS collaboration observed $\gamma$ ray flares from Mrk 421 and found a correlation between $\mathrm{TeV} \gamma$-ray and $X$-ray intensities [24]. An enlarged $\left(\sim 37,000 \mathrm{~m}^{2}\right)$ Tibet-III array started operation in 2003. Using data collected with the Tibet-III array between 1999 and 2005, the measured differential energy spectrum of $\gamma$-rays from the Crab Nebula could be described with a spectral index of $(-2.96 \pm 0.14)$ in the energy range $2-40 \mathrm{TeV}[10]$. This is consistent with data obtained with other experiments, and statistically consistent (within $2.6 \sigma$ ) with a previous Tibet result. No evidence was found for either time variability of flux intensity from the Crab Nebula, or pulsed $\gamma$-rays from the Crab pulsar at multi-TeV energies.

\footnotetext{
${ }^{1}$ The preliminary results for the TeV diffuse flux from the Cygnus region measured with ARGO-YBJ do not show the strong excess reported by the Milagro collaboration at a median energy of $15 \mathrm{TeV}$ [22], and they hypothesize that the difference could be due to the Cygnus Cocoon which had not been discovered at the time of the Milagro measurement.
} 


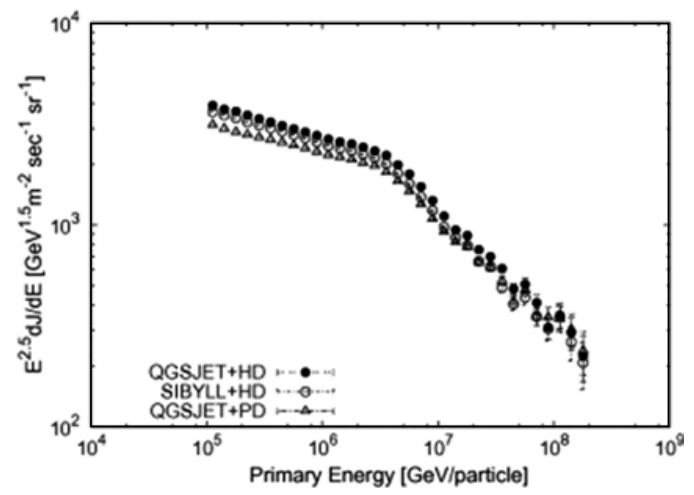

Figure 5. All particle spectrum measured with the Tibet-III array derived with three combinations of hadronic models and mass composition assumptions (QGSJET+HD, SIBYLL+HD and QGSJET+PD). Figure 11 from Ref. [4].

The Tibet collaboration has also measured the energy spectrum of primary cosmic rays in the region of the "knee" [25]. The chemical composition in this region is an important measurement to understand the cosmic-ray acceleration and propagation mechanisms. The primary cosmic-ray nuclei can be identified according to the energy and spatial distributions of the high-energy component of an air shower event detected with the burst detectors and emulsion chambers (at the center of the air shower array). The total energy of the individual $\gamma$-ray air showers is determined by the shower size, which is calculated by fitting the lateral particle density distribution to a given electromagnetic shower theory (a modified NKG structure function). Thus, the energy spectrum of each primary mass group can be determined simultaneously with the burst detectors, emulsion chambers and air shower array. The all-particle spectrum measured with the Tibet-III air-shower array between $10^{14}$ and $10^{17} \mathrm{eV}$ is shown in Fig. 5. Two hadronic interaction models (QGSJET and SIBYLL) are used to estimate the detection efficiency to $\gamma$-rays, and two mass composition models are assumed (proton dominated, PD, and heavy dominated, HD) to estimate the contamination due to heavy primaries. The results shown in Fig. 5 were obtained using the combinations QGSJET+HD, SIBYLL+HD and QGSJET+PD. The systematic uncertainties below the knee are $\sim 20 \%$ due to mass composition, and $\sim 10 \%$ due to hadronic interaction models. The model dependence decreases with energy. The knee of the primary spectrum is observed at an energy of $\sim 4 \mathrm{PeV}$. The measurements of proton and helium spectra with the Tibet experiment suggest the dominance of heavy nuclei around the knee. The sharp knee feature of the spectrum can be explained by either an additional component from a nearby source (or sources) dominated by heavy nuclei, or a nonlinear effect in the diffusive shock acceleration mechanism of cosmic rays that accelerates heavy primaries more efficiently than lighter elements.

\subsection{HAWC}

Once the array of 300 WCDs is completed in early 2015, HAWC will be the most sensitive $\gamma$-ray detector above $10 \mathrm{TeV}$, and will maintain unprecedented wide-field sensitivity to $\gamma$-rays above $100 \mathrm{GeV}$. The differential sensitivity of the HAWC array is shown in the left panel of Fig. 6 together with the sensitivity from other current instruments. The full HAWC array will be an order of magnitude more sensitive than Milagro. Thus, it observes a similar fraction of the sky $\sim 100$ times faster. The sensitivity of the HAWC array to differential $E^{-2}$ spectra 

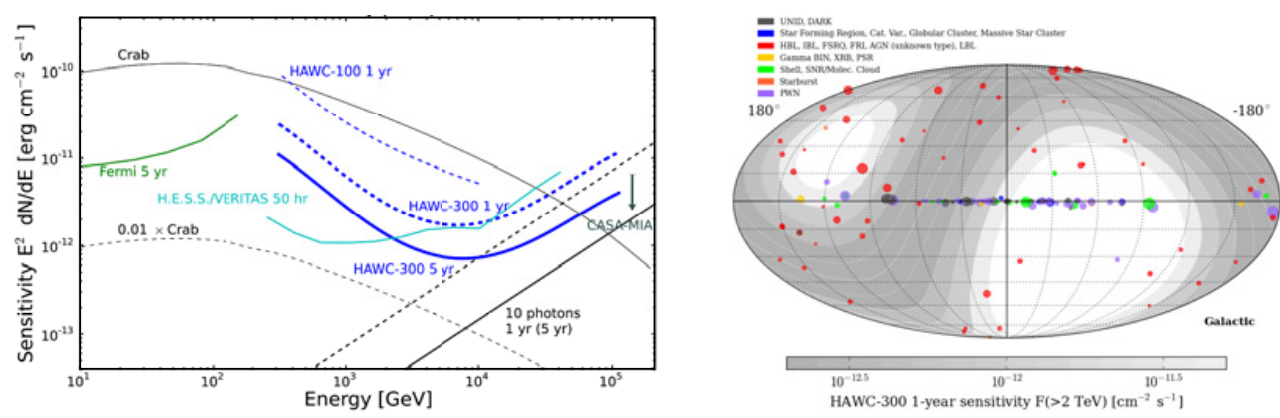

Figure 6. Left: differential sensitivity of HAWC compared to other instruments. (Fig. 4 from Ref. [26].) The sensitivity for the first 100 of the 300 HAWC WCDs is also shown for comparison. Right: sensitivity of the HAWC array to differential $E^{-2}$ spectra along with known sources from the TeVCat TeV catalog [27] in Galactic coordinates. (Fig. 3 from Ref. [26].) The size of the symbols is representative of the flux of the source in the TeVCat catalog.

is shown in the right panel of Fig. 6 in Galactic coordinates along with sources from the TeVCat catalog [27]. The HAWC detector will be sensitive to pure power law spectra at a level of $\sim 50 \mathrm{mCrab}$ above $2 \mathrm{TeV}$ over $5 \mathrm{sr}(40 \%)$ of the sky in one year of exposure. If a source were to flare by a factor of $N$, the time required to see the source at fixed significance would be reduced by $N^{2}$.

With its wide field of view and sensitivity 10 times Milagro's, HAWC will survey the $\gamma$-ray sky measuring Galactic sources beyond $10 \mathrm{TeV}$, and daily monitoring half of the sky for flaring sources. The significance map of the full field of view of HAWC is shown in Fig. 7 using $\gamma$ rays up to $60^{\circ}$ in zenith angle recorded between August, 2013 and June, 2014. (This corresponds to 260 days of live-time.) The number of active WCDs in this period grew from 106 to 133 , and this array is called HAWC-111. The median energy of this map is $\sim 2 \mathrm{TeV}$. This corresponds to roughly one order of magnitude lower energy than Milagro's full sky map (for eight years of data). Maps are smoothed using a Gaussian function with a width proportional to the angular resolution (of the corresponding energy bin). The current angular resolution in the HAWC data is $2.3^{\circ}$ for the lowest energy bin (mean energy $\sim 300 \mathrm{GeV}$ ), and $0.35^{\circ}$ for the highest energy bin (mean energy $\sim 10 \mathrm{TeV}$ ). The energy scale is still preliminary, and this impacts the rate of events that satisfies the event selection. It is important to note that this does not appear to affect the efficiency of the gamma/hadron separation. It is clear that there is some source confusion in certain regions of the sky. The absolute pointing uncertainties and the instrument response are still under investigation.

Both the geometrical reconstruction and the gamma/hadron separation used in the current HAWC analyses have been optimized partly on the observed signal from the Crab Nebula. The significance map of the region around the Crab Nebula is shown in the left panel of Fig. 8. The Crab is observed at a significance level higher than $20 \sigma$ in 260 days of HAWC111. This is consistent with the sensitivity anticipated from simulations after correcting for the angular resolution observed in the HAWC data. This observation is also at the same level of significance of the Milagro detection of the Crab using all their data. It is important to mention that the Crab is observed significantly in all the energy bins. Mrk 421 is also observed in the HAWC-111 data at a significance level of $6.5 \sigma$ (Fig. 7), and there is clear emission from multiple sources along the Galactic plane (right panel of Fig. 8). There is no significant excess observed from the Galactic Center which transits over the HAWC site at a zenith angle of $\sim 48^{\circ}$ at the edge of the HAWC sensitivity. 

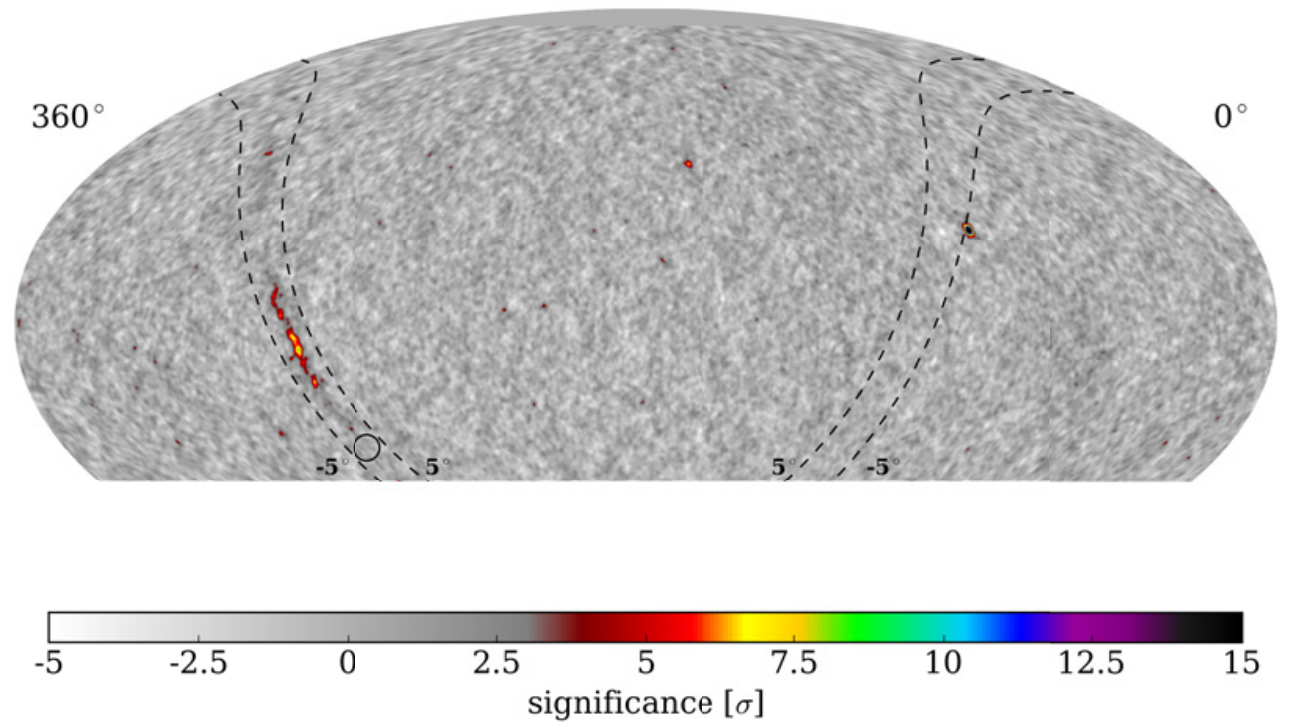

Figure 7. PRELIMINARY. Significance map of the HAWC field of view in equatorial coordinates using 260 days of live-time of HAWC-111. This map includes $\gamma$ rays observed with zenith angles up to $60^{\circ}$. The dashed lines indicate the Galactic latitudes $b= \pm 5^{\circ}$, and the circle marks the location of the Galactic Center.
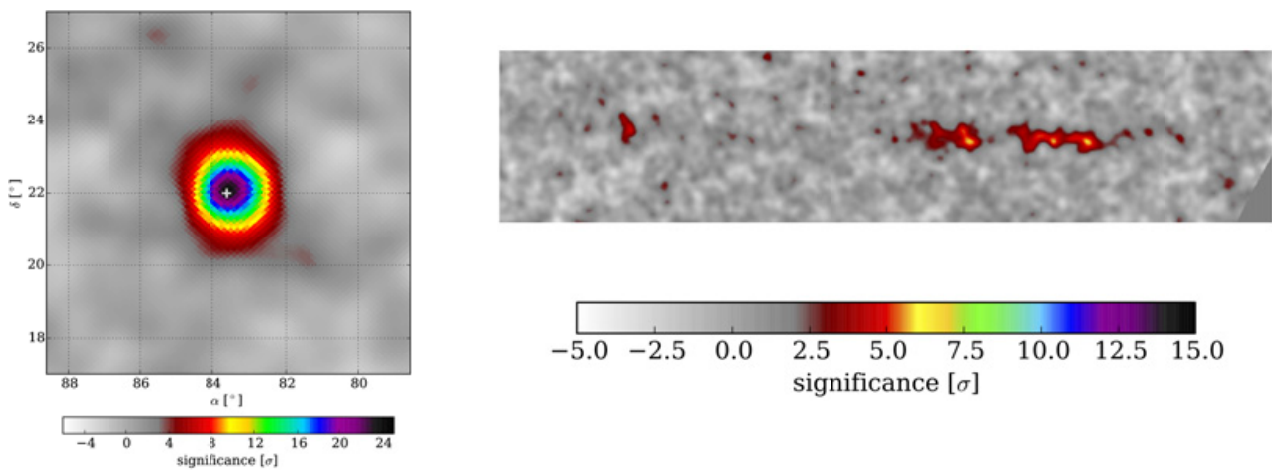

Figure 8. PRELIMINARY. Significance maps using 260 days of live-time of HAWC-111. Left: region around the Crab Nebula. Right: galactic plane $l=\left(0^{\circ}, 90^{\circ}\right), b=\left(-10^{\circ}, 10^{\circ}\right)$.

HAWC data are recorded using two data acquisition (DAQ) systems. The main system is used to record the arrival time and the time over threshold (TOT) of the PMT pulses. This system provides the information necessary to reconstruct the shower core, arrival direction, and lateral distribution of the deposited charge at the ground level. A secondary system, called the scaler DAQ, operates in a simple counting mode, and is sensitive to transient events that produce a sudden increase (or decrease) in the counting rates of the PMTs. Both DAQ systems are sensitive to the high-energy emission from GRBs that extend to at least $30 \mathrm{GeV}$ [28], and have energy thresholds that are low enough to observe events similar to GRB 090510 [29] and GRB 090902b [30] with the characteristics observed by Fermi LAT. While both systems can measure the spectra of GRBs, the sensitivity of the scaler and main DAQ systems to GRBs are complementary. The scaler DAQ reaches lower energies, while the main DAQ 


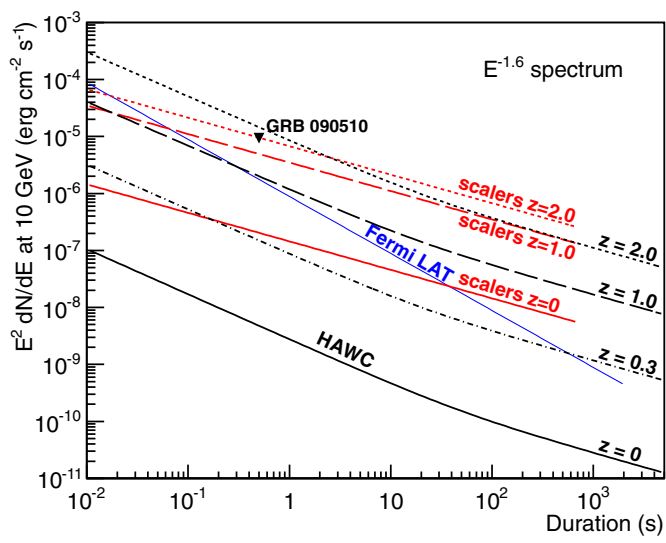

Figure 9. Sensitivity of the HAWC detector to GRBs using the scaler and main DAQ systems as a function of the burst duration. (Fig. 8 from Ref. [28].) The main DAQ uses a simple multiplicity trigger of 70 PMTs or more. The source position is set at a zenith angle of $20^{\circ}$, and the assumed spectrum is $E^{-1.6}$. The sensitivity lines represent the $5 \sigma$ detection level for the scalers (red lines), and the $5 \sigma$ discovery potential for the main DAQ (black lines). The blue line is the flux necessary for the observation of $1 \gamma$-ray above $10 \mathrm{GeV}$ with the Fermi-LAT, and the triangle represents GRB 090510 [29].

can reconstruct the energy and direction of individual $\gamma$-rays. The minimum flux required (for both DAQ systems) to make a $5 \sigma$ detection of a transient source (at a zenith angle of $20^{\circ}$ ) as a function of the burst duration is shown in Fig. 9 assuming a source spectrum of $E^{-1.6}$. The effects of the extragalactic background light (EBL) (modeled as in Ref. [31]) are included for various values of redshift. (These are different for the scalers and main DAQ due to the energy response of each system.) The full HAWC array will be able to detect GRBs with characteristics similar to those already observed with the Fermi-LAT experiment. For example, if a burst such as GRB 090510 were to occur within a zenith angle of $26^{\circ}$, it would be detected as a $5 \sigma$ (or greater) significant excess. Thus, HAWC will be particularly useful to study the high-energy emission of GRBs with hard non-Band power-law spectra.

The detection of $\gamma$-rays with energies above $100 \mathrm{GeV}$ from GRBs is only possible for very bright, nearby bursts because these photons are attenuated in their interactions with the EBL. GRB 130427A is the most energetic burst detected so far with a redshift less than 0.5. Its prompt phase triggered the Fermi-GBM at $T_{0}=07: 47: 06.42$ UTC. The GeV emission was delayed, and between $T_{0}+11.5 \mathrm{~s}$ and $T_{0}+33 \mathrm{~s}$ an additional power-law component was required in the spectral fit. The Fermi-LAT also detected an unprecedented long (about 20 hours, interrupted by occultations) high-energy (above $100 \mathrm{MeV}$ ) emission [32]. This burst also featured the most energetic photon ever detected from a GRB extending the observed energy spectrum to at least $95.3 \mathrm{GeV}$ (128 GeV when corrected for redshift), well within the range accessible to HAWC. At $T_{0}$, only the scaler DAQ was taking data with 29 operational WCDs (called HAWC-30) and 115 PMTs. At that time, the local zenith angle of GRB 130427A was $57^{\circ}$ and setting. The high zenith angle reduces the effective area by 2 orders of magnitude with respect to $20^{\circ}$ for example. (Furthermore, the energy threshold is also significantly higher towards the horizon.) Then, the effective area to bursts overhead for HAWC-30 is also an order of magnitude worse than the full array, HAWC-300. Thus, no statistically significant excess of counts was found and upper limits have been placed [33]. The derived flux upper limits between $20 \mathrm{GeV}$ and $1 \mathrm{TeV}$ using the HAWC effective area for GRB $130427 \mathrm{~A}$ in the $11.5 \mathrm{~s}$ to $33 \mathrm{~s}$ search window are shown in figure 10 . The sensitivity of 


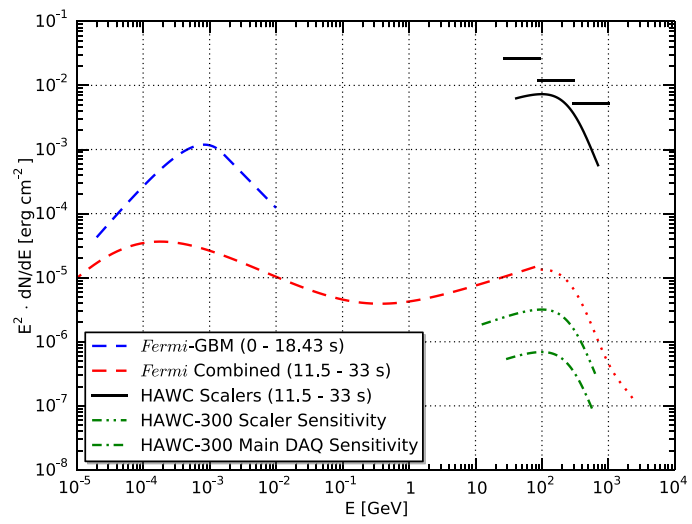

Figure 10. Flux upper limits for GRB 130427A in the 11.5 - $33 \mathrm{~s}$ search window using the effective area of the HAWC array. (Fig. 4 from Ref. [33].) The blue (red) dashed line shows the spectral fit to the prompt GBM (combined GBM and LAT) data. The dotted red line shows the effect of EBL absorption. The black lines show the upper limits for the scaler DAQ assuming the fit to the combined Fermi data and EBL absorption for the integral limit, and assuming a spectrum of $E^{-2}$ for the differential limits. The green dashed-dotted lines show the expected sensitivity to an overhead GRB for the two DAQ systems of the full HAWC array. (The main DAQ is more sensitive, while the scalers reach lower energies.)

the two DAQ systems of the full HAWC-300 array for a GRB within $20^{\circ}$ from the zenith is also shown in the figure (green dashed-dotted lines). It is clear that a similar GRB close to zenith $\left(\theta<26^{\circ}\right)$ would be easily detected by the full HAWC detector. Besides the unusually bright bursts like GRB 130427A, HAWC will be sensitive to other GRBs with a rate as high as one to two per year [34]. Observations with HAWC beyond the Fermi-LAT energy range will constrain the maximum energy produced by the GRB, and/or establish the existence of a cut-off, probing the $\gamma$-ray absorption either in the GRB (probing the bulk Lorentz factor), or along the line of sight due to the extragalactic background light.

While the main design goal of the HAWC observatory is to study $\gamma$-ray sources, the array is also sensitive to charged cosmic rays that are a large background to those $\gamma$-ray searches. But the large number of cosmic rays can also be used to make precise measurements of small deviations from isotropy in the distribution of arrival directions at $\mathrm{TeV}$ energies. This distribution was analyzed using the first $\sim 50$ billion events recorded with HAWC-95 (95 working WCDs) and HAWC-111 between June, 2013 and February, 2014 [35]. This study revealed an anisotropy at the level of $10^{-4}$ on small angular scales $\left(\sim 10^{\circ}\right)$. In a first step, a relative intensity map is obtained by comparing the data to the detector response to an isotropic cosmic-ray flux. Then, the angular power spectrum is determined from the relative intensity map. The map of pre-trial significances is shown in figure 11 after removing the sum of the monopole $(l=0)$, dipole $(l=1)$, quadrupole $(l=2)$, and octupole $(l=3)$ terms. (The lower order multipoles must be removed from the sky map to study the small angular scales.) A smoothing procedure is applied (with a radius of $10^{\circ}$ ) to improve the sensitivity to features on angular scales larger than the pixel size. After the elimination of the large-scale structure, the residual HAWC cosmic-ray sky map shows three regions of significant excess flux. The most significant $(17 \sigma)$ excess is found at $(\alpha, \delta) \approx\left(60^{\circ},-7^{\circ}\right)$, and corresponds to the so-called Region A (Region 1) in the Milagro (ARGO-YBJ) sky map. Even though a detailed study of the energy dependence of the flux in the excess regions is not possible with HAWC at this point, the analysis presented in Ref. [35], using a simple energy proxy based on the number of PMTs in the event and the zenith angle of the cosmic ray, is sufficient to confirm that the energy spectrum of Region A is harder than the isotropic cosmic-ray spectrum. The relative 


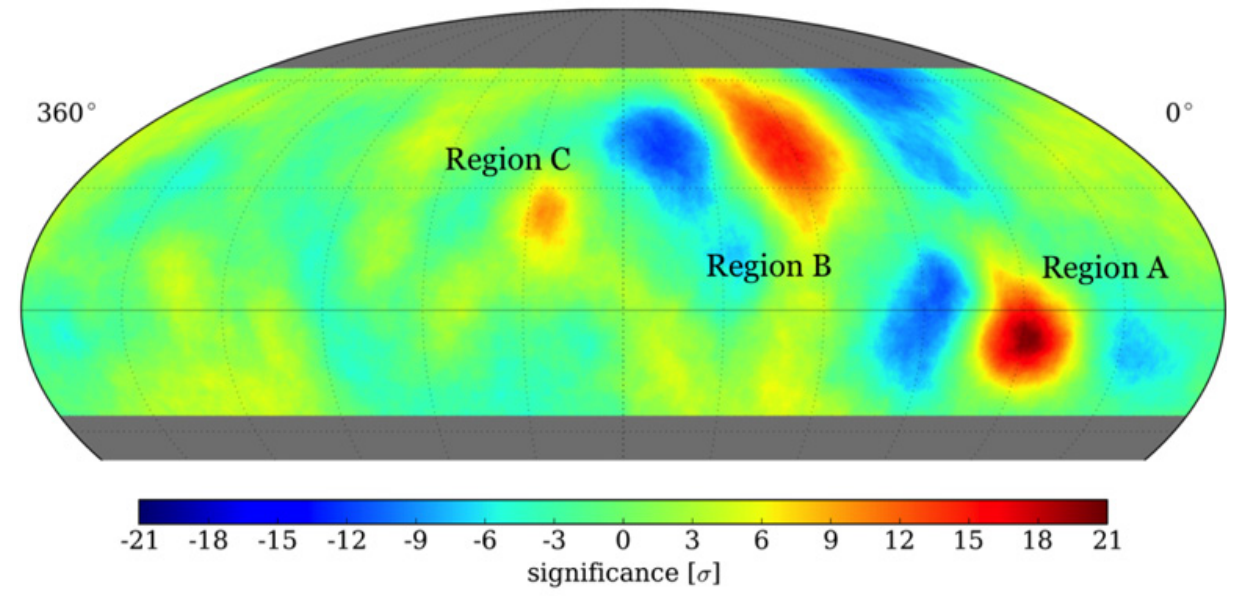

Figure 11. Map of pre-trial significances after subtracting the dipole, quadrupole, and octupole terms from the total cosmic-ray flux. (Fig. 5 from Ref. [35].) There is a $10^{\circ}$ smoothing applied to this map.

intensity of the excess observed with HAWC agrees (within uncertainties) near the location of the centroid of Region A reported by Milagro in the two bins of highest median energy (14 and $19 \mathrm{TeV})$. The second most significant $(11.2 \sigma)$ excess around $\alpha=120^{\circ}$, identified as Region B (Region 2) in the Milagro (ARGO-YBJ) map, is the most extended essentially spanning almost the entire declination range visible to HAWC. The third excess $(8.2 \sigma)$ region, Region C in Fig. 11, is not significant in the Milagro data, but coincides with Region 4 in the ARGO-YBJ map. The median cosmic-ray energy at this declination is $2 \mathrm{TeV}$. With HAWC, this region is detected at almost twice the relative intensity observed by ARGO-YBJ, making it more significant in HAWC despite the considerably shorter observation period. A small excess in the HAWC map near Region 3 in the ARGO-YBJ map is currently not statistically significant $(3.7 \sigma)$. The study of the morphology and relative intensity of all excess regions as a function of energy will be continued with more data in the near future. The full HAWC-300 array will also have an improved energy resolution which will allow for a detailed study of these regions as a function of energy.

Data from the HAWC Observatory will also contribute to tests of Lorentz invariance with transient sources [36], searches for exotic signals such as massive relic particles (SUSY QBalls [37], WIMP dark matter [38]), and primordial black holes [39].

\subsection{LHAASO}

The Large High Altitude Air Shower Observatory (LHAASO) is a proposed project designed to understand the origin of high energy cosmic rays by studying gamma ray sources above $30 \mathrm{TeV}$ [40]. The proposed experiment will cover an area of $1 \mathrm{~km}^{2}$ using five types of detection techniques to measure the arrival direction of air showers, the total number of secondary particles, the muon content, the Cherenkov image, and the high energy gamma rays near the shower core. The proposed layout of LHAASO is shown in Fig. 12.

\subsubsection{LHAASO-KM2A}

The main array of LHAASO will cover an effective area of $1 \mathrm{~km}^{2}$ (KM2A). It will consist of 6,300 scintillator detectors (of $1 \mathrm{~m}^{2}$ each) arranged in a triangular grid with a $15 \mathrm{~m}$ spacing, and 1,220 muon detectors covering an active area of $40,000 \mathrm{~m}^{2}$ with a $30 \mathrm{~m}$ spacing. Each 


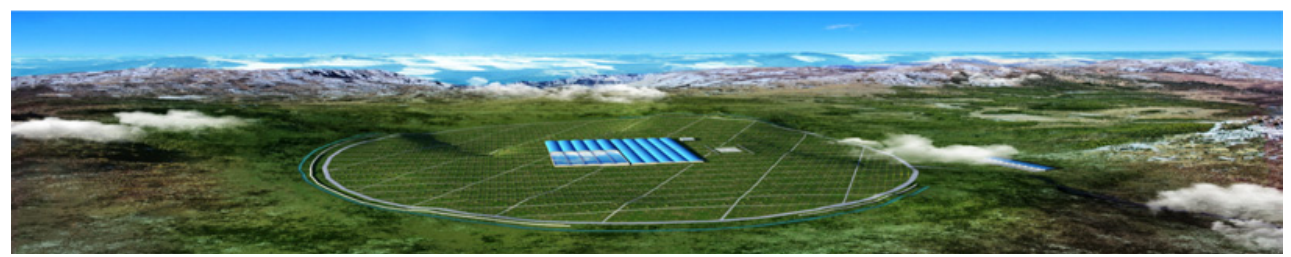

Figure 12. Proposed layout for LHAASO including a $1 \mathrm{~km}^{2}$ main array with 6,300 scintillator and 1,220 muon detectors, a central array with 24 Cherenkov telescopes and 542 burst detectors, and a large water Cherenkov detector $\left(90,000 \mathrm{~m}^{2}\right)$.

electromagnetic particle detector of KM2A will consist of $4 \times 4$ tiles $(25 \mathrm{~cm} \times 25 \mathrm{~cm} \times 2 \mathrm{~cm}$ each) of plastic scintillators. Wavelength-shifting fibers guide the scintillating photons from each group of 16 tiles to a single photomultiplier tube per detector. Each muon detector of $\mathrm{KM} 2 \mathrm{~A}$ is a water Cherenkov detector in a concrete tank ( $3.6 \mathrm{~m}$ in radius and $1.2 \mathrm{~m}$ in height) covered by $2.5 \mathrm{~m}$ of dirt. Each tank will be equipped with one 8" PMT facing downward into a highly reflecting liner bag filled with pure water.

\subsubsection{LHAASO-WCDA}

The design of LHAASO also includes a Water Cherenkov Detector Array (WCDA) covering a total active area of $90,000 \mathrm{~m}^{2}$. The WCDA consists of 4 water ponds, each one of them roughly the size of HAWC $(150 \mathrm{~m} \times 150 \mathrm{~m})$, with a depth of $\sim 4.5 \mathrm{~m}$. Each pond will be subdivided into 900 cells $(30 \times 30$ cells, $5 \mathrm{~m} \times 5 \mathrm{~m}$ each $)$, optically isolated with black plastic curtains that will prevent light contamination from neighboring cells. There will be an upward-looking 8" PMT at the bottom of each cell to collect the Cherenkov light produced by charged particles in the water.

\subsubsection{LHAASO-WFCTA}

Two more detection techniques are proposed for the central array of LHAASO. The additional components are the Wide Field of view Cherenkov Telescope Array (WFCTA) and the high threshold Shower Core Detector Array (SCDA). The 24 wide FOV telescopes of the WFCTA will be designed and arranged to measure the UV light from the air showers observed above the ground array from a distance of $\sim 5 \mathrm{~km}$. There will be telescopes covering elevations from $3^{\circ}$ to $59^{\circ}$ and others covering elevations from $3^{\circ}$ to $31^{\circ}$. Finally, the 24 burst detectors of the SCDA covering an effective area of $5,000 \mathrm{~m}^{2}$ will contribute to the identification of the cosmic-ray species.

\section{Outlook}

The current generation of ground arrays have established very high energy $\gamma$-ray astrophysics as an exciting new window into the electromagnetic spectrum. The large field of view and nearly $100 \%$ duty cycle of ARGO-YBJ, Tibet AS, and HAWC allow surveys of large fractions of the sky and observations in the prompt phase of flaring and transient events. The increased sensitivity of these instruments at energies beyond those covered by satellites have contributed to make the number of identified $\mathrm{TeV}$ sources over one hundred. It is not unreasonable to expect exciting new measurements and discoveries in the near future including $\mathrm{TeV}$ sources with no counterparts at other wavelengths, the study of the acceleration and propagation mechanisms in a wide range of accelerator environments, the identification 
of dark matter, constrains of the extragalactic background radiation, precise measurements of the diffuse background emission, etc.

Each RPC in ARGO-YBJ was instrumented with two large electrodes facing the other side of the gas volume and an analog charge readout over the entire central carpet in December 2009. The detector in this configuration can measure the particle density in the core region from tens to several thousands of particles per $\mathrm{m}^{2}$. The analog readout of the RPCs allows to extend the energy range of the analysis of the existing ARGO-YBJ data to the $1-10 \mathrm{PeV}$ region, and provides highly detailed images of the charged component at the core of those air showers.

A large underground water Cherenkov muon-detector array has been added to the Tibet AS experiment. This array has 80 detectors covering an area of $\sim 4,500 \mathrm{~m}^{2}$, and started taking data in February 2014. Data from this experiment will contribute to the measurement of the differential energy spectra of primary cosmic-ray components in the region of the knee.

The full HAWC array with 300 detectors will start operations in early 2015. With an order of magnitude higher sensitivity with respect to the previous generation of water Cherenkov detectors, HAWC data is expected to significantly contribute to the measurement of extended and large-scale structures (e.g., galactic diffuse emission or the "Fermi bubbles"), the discovery of previously unidentified $\mathrm{TeV}$ sources, the detection of time variable sources of $\gamma$ rays (such as AGN, GRBs, pulsars, and/or binary systems), and the detailed study of the cosmic-ray anisotropy.

Prototypes for the five detection techniques proposed for LHAASO are being tested mostly at the ARGO-YBJ site. Data from LHAASO will expand the searches for very high energy $\gamma$-ray sources beyond the sensitivity of current experiments, and will significantly contribute to our understanding of the origin of cosmic rays, and of the energy spectra and composition of cosmic rays over a wide range of energies.

\section{References}

[1] G. Aielli, et al. [ARGO-YBJ Collaboration], Nucl. Instrum. Meth. A 608, 246 (2009)

[2] G. Aielli, et al. [ARGO-YBJ Collaboration], Nucl. Instrum. Meth. A 661, S56 (2012)

[3] B. Bartoli et al. [ARGO-YBJ Collaboration], ApJ 779, 27 (2013)

[4] M. Amenomori, et al. [Tibet AS Collaboration], Advances in Space Research 47, 629 (2011)

[5] M. Mostafá for the HAWC Collaboration, Proceedings of the 33rd ICRC, Rio de Janeiro, Brazil (2013)

[6] R. Atkins, et al. [Milagro Collaboration], Phys. Rev. Lett. 95, 251103 (2005)

[7] F. Aharonian, et al. [HEGRA Collaboration], ApJ 614, 897 (2004)

[8] F. Aharonian, et al. [H.E.S.S. Collaboration], A\&A 457, 899 (2006)

[9] J. Albert, et al. [MAGIC Collaboration], ApJ 674, 1037 (2008)

[10] M. Amenomori, et al. [Tibet AS Collaboration], ApJ 692, 61 (2009)

[11] B. Bartoli, et al. [ARGO-YBJ Collaboration], ApJ 745, L22 (2012)

[12] A. A. Abdo, et al., ApJ 727, 129 (2011)

[13] B. Bartoli, et al. [ARGO-YBJ Collaboration], ApJ 758, 2 (2012)

[14] B. Bartoli, et al. [ARGO-YBJ Collaboration], ApJ 734, 110 (2011)

[15] A. A. Abdo, et al. [Milagro Collaboration], ApJ 664, L91 (2007)

[16] A. A. Abdo, et al. [Milagro Collaboration], ApJ 753, 159 (2012)

[17] B. Bartoli, et al. [ARGO-YBJ Collaboration], ApJ 790, 152 (2014)

[18] M. Ackermann, et al. [Fermi-LAT Collaboration], Science 334, 1103 (2011)

[19] A. A. Abdo, et al. [Milagro Collaboration], ApJ 658, L33 (2007) 
[20] A. A. Abdo, et al. [Milagro Collaboration], ApJ 664, L91 (2007)

[21] A. A. Abdo, et al. [Milagro Collaboration], ApJ 700, L127 (2009)

[22] A. A. Abdo, et al. [Milagro Collaboration], ApJ 688, 1078 (2008)

[23] G. Aielli, et al. [ARGO-YBJ Collaboration], ApJ 699, 1281 (2009)

[24] M. Amenomori, et al. [Tibet AS Collaboration], ApJ 598, 242 (2003)

[25] M. Amenomori, et al. [Tibet AS Collaboration], Astrophys. Space Sci. Trans. 7, 15 (2011)

[26] A. U. Abeysekara, et al. [HAWC Collaboration], Astroparticle Physics 50-52, 26 (2013)

[27] S. Wakely and D. Horan, http://tevcat.uchicago.edu/.

[28] A. U. Abeysekara, et al. [HAWC Collaboration], Astroparticle Physics 35, 641 (2012)

[29] M. Ackermann, et al. [Fermi-LAT Collaboration], ApJ 716, 1178 (2010)

[30] A. A. Abdo, et al., ApJ 706, L138 (2009)

[31] R. C. Gilmore, et al., MNRAS 399 (2009) 1694-1708

[32] M. Ackermann, et al. [Fermi-LAT Collaboration], Science 343, 42 (2014)

[33] A. U. Abeysekara, et al. [HAWC Collaboration], accepted for publication in ApJ (2015)

[34] I. Taboada, and R. C. Gilmore, Nuclear Instruments and Methods in Physics Research A 742, 276 (2014)

[35] A. U. Abeysekara, et al. [HAWC Collaboration], ApJ 796, 108 (2014)

[36] L. Nellen for the HAWC Collaboration, Proceedings of the 33rd ICRC, Rio de Janeiro, Brazil (2013)

[37] P. Karn for the HAWC Collaboration, Proceedings of the 33rd ICRC, Rio de Janeiro, Brazil (2013)

[38] A. U. Abeysekara, et al. [HAWC Collaboration], Phys. Rev. D 90, 122002 (2014)

[39] A. A. Abdo, et al. [Milagro and HAWC Collaborations], Astroparticle Physics 64, 4 (2015)

[40] Z. Cao and M. Zha for the LHAASO Collaboration, Proceedings of the 33rd ICRC, Rio de Janeiro, Brazil (2013) 